\title{
Rationality of spectral action for Robertson-Walker metrics
}

\author{
Farzad Fathizadeh, Asghar Ghorbanpour and Masoud Khalkhali \\ Department of Mathematics, Middlesex College, Western University, \\ London, Ontario, N6A 5B\%, Canada \\ E-mail: ffathiz@uwo.ca, aghorba@uwo.ca, masoud@uwo.ca
}

ABstract: We use pseudodifferential calculus and heat kernel techniques to prove a conjecture by Chamseddine and Connes on rationality of the coefficients of the polynomials in the cosmic scale factor $a(t)$ and its higher derivatives, which describe the general terms $a_{2 n}$ in the expansion of the spectral action for general Robertson-Walker metrics. We also compute the terms up to $a_{12}$ in the expansion of the spectral action by our method. As a byproduct, we verify that our computations agree with the terms up to $a_{10}$ that were previously computed by Chamseddine and Connes by a different method.

KEYwords: Non-Commutative Geometry, Models of Quantum Gravity

ARXIV EPRINT: 1407.5972 


\section{Contents}

1 Introduction 1

2 The Dirac operator for Robertson-Walker metrics 3

2.1 Levi-Civita connection 3

2.2 The spin connection of Robertson-Walker metrics in Hopf coordinates 4

2.3 The Dirac operator of Robertson-Walker metrics in Hopf coordinates 5

3 Terms up to $a_{10}$ and their agreement with Chamseddine-Connes' result 6

3.1 Small time heat kernel expansions using pseudodifferential calculus 6

$\begin{array}{lll}3.2 & \text { The terms up to } a_{6} & 9\end{array}$

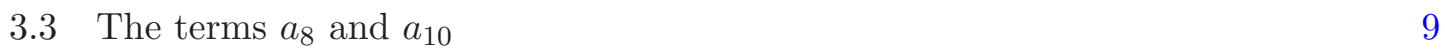

4 Computation of the term $a_{12}$ in the expansion of the spectral action $\quad 10$

4.1 The result of the computation in Hopf coordinates 10

4.2 Agreement of the result with computations in spherical coordinates 12

$\begin{array}{lll}4.3 & \text { Agreement with the full expansion for the round metric } & 13\end{array}$

5 Chameseddine-Connes' conjecture $\quad \mathbf{1 3}$

5.1 Proof of rationality of the coefficients in the expressions for $a_{2 n} \quad 13$

5.2 A recursive formula for the coefficient of the highest order term in $a_{2 n} \quad 17$

$\begin{array}{llr}6 & \text { Conclusions } & 18\end{array}$

\section{Introduction}

Noncommutative geometry in the sense of Alain Connes [11] has provided a paradigm for geometry in the noncommutative setting based on spectral data. This generalizes Riemannian geometry [14] and incorporates physical models of elementary particle physics [5, $7,10,12,13,15,19,32-34]$. An outstanding feature of the spectral action defined for noncommutative geometries is that it derives the Lagrangian of the physical models from simple noncommutative geometric data $[4,10,13]$. Thus, various methods have been developed for computing the terms in the expansion in the energy scale $\Lambda$ of the spectral action $[3,6,8,9,20,21]$. Potential applications of noncommutative geometry in cosmology have recently been carried out in [16, 22, 25-31].

Noncommutative geometric spaces are described by spectral triples $(\mathcal{A}, \mathcal{H}, D)$, where $\mathcal{A}$ is an involutive algebra represented by bounded operators on a Hilbert space $\mathcal{H}$, and $D$ is an unbounded self-adjoint operator acting in $\mathcal{H}$ [11]. The operator $D$, which plays the role of the Dirac operator, encodes the metric information and it is further assumed that it has bounded commutators with elements of $\mathcal{A}$. It has been shown that if $\mathcal{A}$ is commutative and 
the triple satisfies suitable regularity conditions then $\mathcal{A}$ is the algebra of smooth functions on a $\operatorname{spin}^{c}$ manifold $M$ and $D$ is the Dirac operator acting in the Hilbert space of $L^{2}$ spinors [14]. In this case, the Seeley-de Witt coefficients $a_{n}\left(D^{2}\right)=\int_{M} a_{n}\left(x, D^{2}\right) d v(x)$, which vanish for odd $n$, appear in a small time asymptotic expansion of the form

$$
\operatorname{Tr}\left(e^{-t D^{2}}\right) \sim t^{-\operatorname{dim}(M) / 2} \sum_{n \geq 0} a_{2 n}\left(D^{2}\right) t^{n} \quad(t \rightarrow 0) .
$$

These coefficients determine the terms in the expansion of the spectral action. That is, there is an expansion of the form

$$
\operatorname{Tr} f\left(D^{2} / \Lambda^{2}\right) \sim \sum_{n \geq 0} f_{2 n} a_{2 n}\left(D^{2} / \Lambda^{2}\right),
$$

where $f$ is a positive even function defined on the real line, and $f_{2 n}$ are the moments of the function $f[3,4]$. See Theorem 1.145 in [15] for details in a more general setup, namely for spectral triples with simple dimension spectrum.

By devising a direct method based on the Euler-Maclaurin formula and the FeynmanKac formula, Chamseddine and Connes have initiated in [9] a detailed study of the spectral action for the Robertson-Walker metric with a general cosmic scale factor $a(t)$. They calculated the terms up to $a_{10}$ in the expansion and checked the agreement of the terms up to $a_{6}$ against Gilkey's universal formulas $[17,18]$.

The present paper is intended to compute the term $a_{12}$ in the spectral action for general Robertson-Walker metrics, and to prove the conjecture of Chamseddine and Connes [9] on rationality of the coefficients of the polynomials in $a(t)$ and its derivatives that describe the general terms $a_{2 n}$ in the expansion. In passing, we compare the outcome of our computations up to the term $a_{10}$ with the expressions obtained in [9], and confirm their agreement.

In terms of the above aims, explicit formulas for the Dirac operator of the RobertsonWalker metric and its pseudodifferential symbol in Hopf coordinates are derived in section 2. Following a brief review of the heat kernel method for computing local invariants of elliptic differential operators using pseudodifferential calculus [17], we compute in section 3 the terms up to $a_{10}$ in the expansion of the spectral action for Robertson-Walker metrics. The outcome of our calculations confirms the expressions obtained in [9]. This forms a check in particular on the validity of $a_{8}$ and $a_{10}$, which as suggested in [9] also, seems necessary due to the high complexity of the formulas. In section 4, we record the expression for the term $a_{12}$ achieved by a significantly heavier computation, compared to the previous terms. It is checked that the reduction of $a_{12}$ to the round case $a(t)=\sin t$ conforms to the full expansion obtained in [9] for the round metric by remarkable calculations that are based on the Euler-Maclaurin formula. In order to validate our expression for $a_{12}$, parallel but completely different computations are performed in spherical coordinates and the final results are confirmed to match precisely with our calculations in Hopf coordinates.

In section 5, we prove the conjecture made in [9] on rationality of the coefficients appearing in the expressions for the terms of the spectral action for Robertson-Walker metrics. That is, we show that the term $a_{2 n}$ is of the form $Q_{2 n}\left(a(t), a^{\prime}(t), \ldots, a^{(2 n)}(t)\right) / a(t)^{2 n-3}$, where $Q_{2 n}$ is a polynomial with rational coefficients. We also find a formula for the coefficient of the term with the highest derivate of $a(t)$ in $a_{2 n}$. It is known that values of Feynman 
integrals for quantum gauge theories are closely related to multiple zeta values and periods in general and hence tend to be transcendental numbers [24]. In sharp distinction, the rationality result proved in this paper is valid for all scale factors $a(t)$ in Robertson-Walker metrics. Although it might be exceedingly difficult, it is certainly desirable to find all the terms $a_{2 n}$ in the spectral action. The rationality result is a consequence of a certain symmetry in the heat kernel and it is plausible that this symmetry would eventually reveal the full structure of the coefficients $a_{2 n}$. This is a task for a future work. Our main conclusions are summarized in section 6 .

\section{The Dirac operator for Robertson-Walker metrics}

According to the spectral action principle [4, 12], the spectral action of any geometry depends on its Dirac operator since the terms in the expansion are determined by the high frequency behavior of the eigenvalues of this operator. For spin manifolds, the explicit computation of the Dirac operator in a coordinate system is most efficiently achieved by writing its formula after lifting the Levi-Civita connection on the cotangent bundle to the spin connection on the spin bundle. In this section, we summarize this formalism and compute the Dirac operator of the Robertson-Walker metric in Hopf coordinates. Throughout this paper we use Einstein's summation convention without any further notice.

\subsection{Levi-Civita connection}

The spin connection of any spin manifold $M$ is the lift of the Levi-Civita connection for the cotangent bundle $T^{*} M$ to the spin bundle. Let us, therefore, recall the following recipe for computing the Levi-Civita connection and thereby the spin connection of $M$. Given an orthonormal frame $\left\{\theta_{\alpha}\right\}$ for the tangent bundle $T M$ and its dual coframe $\left\{\theta^{\alpha}\right\}$, the connection 1-forms $\omega_{\beta}^{\alpha}$ of any connection $\nabla$ on $T^{*} M$ are defined by

$$
\nabla \theta^{\alpha}=\omega_{\beta}^{\alpha} \theta^{\beta}
$$

Since the Levi-Civita connection is the unique torsion free connection which is compatible with the metric, its 1 -forms are uniquely determined by

$$
d \theta^{\beta}=\omega_{\alpha}^{\beta} \wedge \theta^{\alpha}
$$

This is justified by the fact that the compatibility with metric enforces the relations

$$
\omega_{\beta}^{\alpha}=-\omega_{\alpha}^{\beta},
$$

while, taking advantage of the first Cartan structure equation, the torsion-freeness amounts to the vanishing of

$$
T^{\alpha}=d \theta^{\alpha}-\omega_{\beta}^{\alpha} \wedge \theta^{\beta}
$$




\subsection{The spin connection of Robertson-Walker metrics in Hopf coordinates}

The (Euclidean) Robertson-Walker metric with the cosmic scale factor $a(t)$ is given by

$$
d s^{2}=d t^{2}+a^{2}(t) d \sigma^{2},
$$

where $d \sigma^{2}$ is the round metric on the 3 -sphere $\mathbb{S}^{3}$. It is customary to write this metric in spherical coordinates, however, for our purposes which will be explained below, it is more convenient to use the Hopf coordinates, which parametrize the 3 -sphere $S^{3} \subset \mathbb{C}^{2}$ by

$$
z_{1}=e^{i \phi_{1}} \sin (\eta), \quad z_{2}=e^{i \phi_{2}} \cos (\eta)
$$

with $\eta$ ranging in $[0, \pi / 2)$ and $\phi_{1}, \phi_{2}$ ranging in $[0,2 \pi)$. The Robertson-Walker metric in the coordinate system $x=\left(t, \eta, \phi_{1}, \phi_{2}\right)$ is thus given by

$$
d s^{2}=d t^{2}+a^{2}(t)\left(d \eta^{2}+\sin ^{2}(\eta) d \phi_{1}^{2}+\cos ^{2}(\eta) d \phi_{2}^{2}\right) .
$$

An orthonormal coframe for $d s^{2}$ is then provided by

$$
\theta^{1}=d t, \quad \theta^{2}=a(t) d \eta, \quad \theta^{3}=a(t) \sin \eta d \phi_{1}, \quad \theta^{4}=a(t) \cos \eta d \phi_{2} .
$$

Applying the exterior derivative to these forms, one can easily show that they satisfy the following equations, which determine the connection 1-forms of the Levi-Civita connection:

$$
\begin{aligned}
d \theta^{1} & =0, \\
d \theta^{2} & =\frac{a^{\prime}(t)}{a(t)} \theta^{1} \wedge \theta^{2}, \\
d \theta^{3} & =\frac{a^{\prime}(t)}{a(t)} \theta^{1} \wedge \theta^{3}+\frac{\cot \eta}{a(t)} \theta^{2} \wedge \theta^{3}, \\
d \theta^{4} & =\frac{a^{\prime}(t)}{a(t)} \theta^{1} \wedge \theta^{4}-\frac{\tan \eta}{a(t)} \theta^{2} \wedge \theta^{4} .
\end{aligned}
$$

We recast the above equations into the matrix of connection 1-forms

$$
\omega=\frac{1}{a(t)}\left(\begin{array}{cccc}
0 & -a^{\prime}(t) \theta^{2} & -a^{\prime}(t) \theta^{3} & -a^{\prime}(t) \theta^{4} \\
a^{\prime}(t) \theta^{2} & 0 & -\cot \eta \theta^{3} & \tan \eta \theta^{4} \\
a^{\prime}(t) \theta^{3} & \cot \eta \theta^{3} & 0 & 0 \\
a^{\prime}(t) \theta^{4} & -\tan \eta \theta^{4} & 0 & 0
\end{array}\right) \in \mathfrak{s o}(4),
$$

which lifts to the spin bundle using the Lie algebra isomorphism $\mu: \mathfrak{s o}(4) \rightarrow \mathfrak{s p i n}(4)$ given by (see [23])

$$
\mu(A)=\frac{1}{4} \sum_{\alpha, \beta}\left\langle A \theta^{\alpha}, \theta^{\beta}\right\rangle c\left(\theta^{\alpha}\right) c\left(\theta^{\beta}\right), \quad A \in \mathfrak{s o}(4) .
$$

Since $\left\langle\omega \theta^{\alpha}, \theta^{\beta}\right\rangle=\omega_{\beta}^{\alpha}$, the lifted connection $\tilde{\omega}$ is written as

$$
\tilde{\omega}=\frac{1}{4} \sum_{\alpha, \beta} \omega_{\beta}^{\alpha} c\left(\theta^{\alpha}\right) c\left(\theta^{\beta}\right)
$$


In the case of the Robertson-Walker metric we find that

$$
\tilde{\omega}=\frac{1}{2 a(t)}\left(a^{\prime}(t) \theta^{2} \gamma^{12}+a^{\prime}(t) \theta^{3} \gamma^{13}+a^{\prime}(t) \theta^{4} \gamma^{14}+\cot (\eta) \theta^{3} \gamma^{23}-\tan (\eta) \theta^{4} \gamma^{24}\right),
$$

where we use the notation $\gamma^{i j}=\gamma^{i} \gamma^{j}$ for products of pairs of the gamma matrices $\gamma^{1}, \gamma^{2}, \gamma^{3}, \gamma^{4}$, which are respectively written as

$$
\left(\begin{array}{cccc}
0 & 0 & i & 0 \\
0 & 0 & 0 & i \\
i & 0 & 0 & 0 \\
0 & i & 0 & 0
\end{array}\right),\left(\begin{array}{cccc}
0 & 0 & 0 & 1 \\
0 & 0 & 1 & 0 \\
0 & -1 & 0 & 0 \\
-1 & 0 & 0 & 0
\end{array}\right),\left(\begin{array}{cccc}
0 & 0 & 0 & -i \\
0 & 0 & i & 0 \\
0 & i & 0 & 0 \\
-i & 0 & 0 & 0
\end{array}\right),\left(\begin{array}{cccc}
0 & 0 & 1 & 0 \\
0 & 0 & 0 & -1 \\
-1 & 0 & 0 & 0 \\
0 & 1 & 0 & 0
\end{array}\right) .
$$

\subsection{The Dirac operator of Robertson-Walker metrics in Hopf coordinates}

Using the expression (2.1) obtained for the spin connection and considering the predual of the orthonormal coframe $\left\{\theta^{\alpha}\right\}$,

$$
\theta_{1}=\frac{\partial}{\partial t}, \quad \theta_{2}=\frac{1}{a(t)} \frac{\partial}{\partial \eta}, \quad \theta_{3}=\frac{1}{a(t) \sin \eta} \frac{\partial}{\partial \phi_{1}}, \quad \theta_{4}=\frac{1}{a(t) \cos \eta} \frac{\partial}{\partial \phi_{2}}
$$

we compute the Dirac operator for the Robertson-Walker metric explicitly:

$$
\begin{aligned}
D= & c\left(\theta^{\alpha}\right) \nabla_{\theta_{\alpha}} \\
= & \gamma^{\alpha}\left(\theta_{\alpha}+\tilde{\omega}\left(\theta_{\alpha}\right)\right) \\
= & \gamma^{1}\left(\frac{\partial}{\partial t}\right)+\gamma^{2}\left(\frac{1}{a} \frac{\partial}{\partial \eta}+\frac{a^{\prime}}{2 a} \gamma^{12}\right)+\gamma^{3}\left(\frac{1}{a \sin (\eta)} \frac{\partial}{\partial \phi_{1}}+\frac{a^{\prime}}{2 a} \gamma^{13}+\frac{\cot (\eta)}{2 a} \gamma^{23}\right) \\
& +\gamma^{4}\left(\frac{1}{a \cos (\eta)} \frac{\partial}{\partial \phi_{2}}+\frac{a^{\prime}}{2 a} \gamma^{14}-\frac{\tan (\eta)}{2 a} \gamma^{24}\right) \\
= & \gamma^{1} \frac{\partial}{\partial t}+\gamma^{2} \frac{1}{a} \frac{\partial}{\partial \eta}+\gamma^{3} \frac{1}{a \sin \eta} \frac{\partial}{\partial \phi_{1}}+\gamma^{4} \frac{1}{a \cos \eta} \frac{\partial}{\partial \phi_{2}}+\frac{3 a^{\prime}}{2 a} \gamma^{1}+\frac{\cot (2 \eta)}{a} \gamma^{2} .
\end{aligned}
$$

Thus the pseudodifferential symbol of $D$ is given by

$$
\sigma_{D}(x, \xi)=i \xi_{1} \gamma^{1}+\frac{i \xi_{2}}{a} \gamma^{2}+\frac{i \xi_{3}}{a \sin \eta} \gamma^{3}+\frac{i \xi_{4}}{a \cos \eta} \gamma^{4}+\frac{3 a^{\prime}}{2 a} \gamma^{1}+\frac{\cot (2 \eta)}{a} \gamma^{2} .
$$

For the purpose of employing pseudodifferential calculus in the sequel to compute the heat coefficients, we record in the following proposition the pseudodifferential symbol of $D^{2}$. This can be achieved by a straightforward computation to find an explicit expression for $D^{2}$, or alternatively, one can apply the composition rule for symbols, $\sigma_{P_{1} P_{2}}(x, \xi)=$ $\sum_{\alpha} \frac{(-i)^{|\alpha|}}{\alpha !} \partial_{\xi}^{\alpha} \sigma_{P_{1}} \partial_{x}^{\alpha} \sigma_{P_{2}}$, to the symbol of $D$.

Proposition 1. The pseudodifferential symbol of $D^{2}$, where $D$ is the Dirac operator for the Robertson-Walker metric, is given by

$$
\sigma\left(D^{2}\right)=p_{2}+p_{1}+p_{0},
$$

where the homogeneous components $p_{i}$ of order $i$ are written as

$$
p_{2}=\xi_{1}^{2}+\frac{1}{a^{2}} \xi_{2}^{2}+\frac{1}{a^{2} \sin ^{2}(\eta)} \xi_{3}^{2}+\frac{1}{a^{2} \cos ^{2}(\eta)} \xi_{4}^{2},
$$




$$
\begin{aligned}
p_{1}= & \frac{-3 i a a^{\prime}}{a^{2}} \xi_{1}+\frac{-i a^{\prime} \gamma^{12}-2 i \cot (2 \eta)}{a^{2}} \xi_{2}-\frac{i a^{\prime} \csc (\eta) \gamma^{13}+i \cot (\eta) \csc (\eta) \gamma^{23}}{a^{2}} \xi_{3} \\
& +\frac{i \tan (\eta) \sec (\eta) \gamma^{24}-i a^{\prime} \sec (\eta) \gamma^{14}}{a^{2}} \xi_{4} \\
p_{0}= & \frac{1}{4 a(t)^{2}}\left(-6 a(t) a^{\prime \prime}(t)-3 a^{\prime}(t)^{2}+\csc ^{2}(\eta)+\sec ^{2}(\eta)\right. \\
& \left.+4+2 a^{\prime}(t)(\cot (\eta)-\tan (\eta)) \gamma^{12}\right) .
\end{aligned}
$$

\section{Terms up to $a_{10}$ and their agreement with Chamseddine-Connes' result}

The computation of the terms in the expansion of the spectral action for a spin manifold, or equivalently the calculation of the heat coefficients, can be achieved by recursive formulas while working in the heat kernel scheme of local invariants of elliptic differential operators and index theory [17]. Pseudodifferential calculus is an effective tool for dealing with the necessary approximations for deriving the small time asymptotic expansions in which the heat coefficients appear. Universal formulas in terms of the Riemann curvature operator and its contractions and covariant derivatives are written in the literature only for the terms up to $a_{10}$, namely Gilkey's formulas up to $a_{6}[17,18]$ and the formulas in $[1,2,35]$ for $a_{8}$ and $a_{10}$.

\subsection{Small time heat kernel expansions using pseudodifferential calculus}

In [17], by appealing to the Cauchy integral formula and using pseudodifferential calculus, recursive formulas for the heat coefficients of elliptic differential operators are derived. That is, one writes ${ }^{1}$

$$
e^{-t D^{2}}=-\frac{1}{2 \pi i} \int_{\gamma} e^{-t \lambda}\left(D^{2}-\lambda\right)^{-1} d \lambda
$$

where the contour $\gamma$ goes around the non-negative real axis in the counterclockwise direction, and one uses pseudodifferential calculus to approximate $\left(D^{2}-\lambda\right)^{-1}$ via the homogeneous terms appearing in the expansion of the symbol of the parametrix of $D^{2}-\lambda$. Although left and right parametrices have the same homogeneous components, for the purpose of finding recursive formulas for the coefficients appearing in each component, which will be explained shortly, it is more convenient for us to consider the right parametrix $\tilde{R}(\lambda)$. Therefore, the next task is to compute recursively the homogeneous pseudodifferential symbols $r_{j}$ of order $-2-j$ in the expansion of $\sigma(\tilde{R}(\lambda))$. Using the calculus of symbols, with the crucial nuance that $\lambda$ is considered to be of order 2 , one finds that

$$
r_{0}=\left(p_{2}-\lambda\right)^{-1},
$$

\footnotetext{
${ }^{1}$ Hereafter in this paper $t$ denotes the first variable of the space when it appears in $a(t)$ and its derivatives and it denotes the time when it appears in the heat operator and the associated small time asymptotic expansions.
} 
and for any $n>1$

$$
r_{n}=-r_{0} \sum_{\substack{j+2-k=n \\|\alpha|+n}} \frac{(-i)^{|\alpha|}}{\alpha !} d_{\xi}^{\alpha} p_{k} d_{x}^{\alpha} r_{j} .
$$

We summarize the process of obtaining the heat coefficients by explaining that one then uses these homogeneous terms in the Cauchy integral formula to approximate the integral kernel of $e^{-t D^{2}}$. Integration of the kernel of this operator on the diagonal yields a small time asymptotic expansion of the form

$$
\operatorname{Tr}\left(e^{-t D^{2}}\right) \sim \sum_{n=0}^{\infty} \frac{t^{(n-4) / 2}}{16 \pi^{4}} \int \operatorname{tr}\left(e_{n}(x)\right) d v o l_{g} \quad(t \rightarrow 0),
$$

where

$$
e_{n}(x) \sqrt{\operatorname{det} g}=\frac{-1}{2 \pi i} \iint_{\gamma} e^{-\lambda} r_{n}(x, \xi, \lambda) d \lambda d \xi
$$

For detailed discussions, we refer the reader to [17].

It is clear from (2.2) that cross derivatives of $p_{2}$ vanish and $d_{\xi}^{\alpha} p_{k}=0$ if $|\alpha|>k$. Furthermore, $\frac{\partial}{\partial \phi_{k}} r_{n}=0$ for $n \geq 0$, and the summation (3.1) is written as

$$
\begin{aligned}
r_{n}= & -r_{0} p_{0} r_{n-2}-r_{0} p_{1} r_{n-1}+i r_{0} \frac{\partial}{\partial \xi_{1}} p_{1} \frac{\partial}{\partial t} r_{n-2}+i r_{0} \frac{\partial}{\partial \xi_{2}} p_{1} \frac{\partial}{\partial \eta} r_{n-2} \\
& +i r_{0} \frac{\partial}{\partial \xi_{1}} p_{2} \frac{\partial}{\partial t} r_{n-1}+i r_{0} \frac{\partial}{\partial \xi_{2}} p_{2} \frac{\partial}{\partial \eta} r_{n-1}+\frac{1}{2} r_{0} \frac{\partial^{2}}{\partial \xi_{1}^{2}} p_{2} \frac{\partial^{2}}{\partial t^{2}} r_{n-2} \\
& +\frac{1}{2} r_{0} \frac{\partial^{2}}{\partial \xi_{2}^{2}} p_{2} \frac{\partial^{2}}{\partial \eta^{2}} r_{n-2} .
\end{aligned}
$$

Using induction, we find that

$$
r_{n}=\sum_{\substack{2 j-2-|\alpha|=n \\ n / 2+1 \leq j \leq 2 n+1}} r_{n, j, \alpha}(x) r_{0}^{j} \xi^{\alpha} .
$$

For example, one can see that for $n=0$ the only non-zero $r_{0, j, \alpha}$ is $r_{0,1,0}=1$, and for $n=1$ the non-vanishing terms are

$$
r_{1,2, \mathbf{e}_{k}}=\frac{\partial p_{1}}{\partial \xi_{k}}, \quad r_{1,3,2 \mathbf{e}_{l}+\mathbf{e}_{k}}=-2 i g^{k k} \frac{\partial g^{l l}}{\partial x_{k}},
$$

where $\mathbf{e}_{j}$ denotes the $j$-th standard unit vector in $\mathbb{R}^{4}$.

It then follows from the equations (3.2), (3.3) and (3.4) that

$$
\begin{aligned}
e_{n}(x) a(t)^{3} \sin (\eta) \cos (\eta) & =\frac{-1}{2 \pi i} \int_{\mathbb{R}^{4}} \int_{\gamma} e^{-t \lambda} r_{n}(x, \xi, \lambda) d \lambda d \xi \\
& =\sum r_{n, j, \alpha}(x) \int_{\mathbb{R}^{4}} \xi^{\alpha} \frac{-1}{2 \pi i} \int_{\gamma} e^{-t \lambda} r_{0}^{j} d \lambda d \xi
\end{aligned}
$$




$$
=\sum \frac{c_{\alpha}}{(j-1) !} r_{n, j, \alpha} a(t)^{\alpha_{2}+\alpha_{3}+\alpha_{4}+3} \sin (\eta)^{\alpha_{3}+1} \cos (\eta)^{\alpha_{4}+1},
$$

where

$$
c_{\alpha}=\prod_{k} \Gamma\left(\frac{\alpha_{k}+1}{2}\right) \frac{(-1)^{\alpha_{k}}+1}{2} .
$$

It is straightforward to justify the latter using these identities:

$$
\begin{aligned}
\frac{1}{2 \pi i} \int_{\gamma} e^{-\lambda} r_{0}^{j} d \lambda & =(-1)^{j} \frac{(-1)^{j-1}}{(j-1) !} e^{-\|\xi\|^{2}}=\frac{-1}{(j-1) !} \prod_{k=1}^{4} e^{-g^{k k} \xi_{k}^{2}} \\
\int_{\mathbb{R}} x^{n} e^{-b x^{2}} d x & =\frac{1}{2}\left((-1)^{n}+1\right) b^{-\frac{n}{2}-\frac{1}{2}} \Gamma\left(\frac{n+1}{2}\right) .
\end{aligned}
$$

A key point that facilitates our calculations and the proof of our main theorem presented in section 5.1 is the derivation of recursive formulas for the coefficients $r_{n, j, \alpha}$ as follows. By substitution of (3.4) into (3.3) we find a recursive formula of the form

$$
\begin{aligned}
r_{n, j, \alpha}=- & p_{0} r_{n-2, j-1, \alpha}-\sum_{k} \frac{\partial p_{1}}{\partial \xi_{k}} r_{n-1, j-1, \alpha-\mathbf{e}_{k}} \\
& +i \sum_{k} \frac{\partial p_{1}}{\partial \xi_{k}} \frac{\partial}{\partial x_{k}} r_{n-2, j-1, \alpha}+i(2-j) \sum_{k, l} \frac{\partial g^{l l}}{\partial x_{k}} \frac{\partial p_{1}}{\partial \xi_{k}} r_{n-2, j-2, \alpha-2 \mathbf{e}_{l}} \\
& +2 i \sum_{k} g^{k k} \frac{\partial}{\partial x_{k}} r_{n-1, j-1, \alpha-\mathbf{e}_{k}}+i(4-2 j) \sum_{k, l} g^{k k} \frac{\partial g^{l l}}{\partial x_{k}} r_{n-1, j-2, \alpha-2 \mathbf{e}_{l}-\mathbf{e}_{k}} \\
& +\sum_{k} g^{k k} \frac{\partial^{2}}{\partial x_{k}^{2}} r_{n-2, j-1, \alpha}+(4-2 j) \sum_{k, l} g^{k k} \frac{\partial g^{l l}}{\partial x_{k}} \frac{\partial}{\partial x_{k}} r_{n-2, j-2, \alpha-2 \mathbf{e}_{l}} \\
& +(2-j) \sum_{k, l} g^{k k} \frac{\partial^{2} g^{l l}}{\partial x_{k}^{2}} r_{n-2, j-2, \alpha-2 \mathbf{e}_{l}} \\
& +(3-j)(2-j) \sum_{k, l, l^{\prime}} g^{k k} \frac{\partial g^{l l}}{\partial x_{k}} \frac{\partial g^{l^{\prime} l^{\prime}}}{\partial x_{k}} r_{n-2, j-3, \alpha-2 \mathbf{e}_{l}-2 \mathbf{e}_{l^{\prime}}} .
\end{aligned}
$$

It is undeniable that the mechanism described above for computing the heat coefficients involves heavy computations which need to be overcome by computer programming. Calculating explicitly the functions $e_{n}(x), n=0,2, \ldots, 12$, and computing their integrals over $\mathbb{S}_{a}^{3}$ with computer assistance, we find the explicit polynomials in $a(t)$ and its derivatives recorded in the sequel, which describe the corresponding terms in the expansion of the spectral action for the Robertson-Walker metric. That is, each function $a_{n}$ recorded below is the outcome of

$$
\begin{aligned}
a_{n} & =\frac{1}{16 \pi^{4}} \int_{\mathbb{S}_{a}^{3}} \operatorname{tr}\left(e_{n}\right) d v o l_{g} \\
& =\frac{1}{16 \pi^{4}} \int_{0}^{2 \pi} \int_{0}^{2 \pi} \int_{0}^{\pi / 2} \operatorname{tr}\left(e_{n}\right) a(t)^{3} \sin (\eta) \cos (\eta) d \eta d \phi_{1} d \phi_{2} .
\end{aligned}
$$




\subsection{The terms up to $a_{6}$}

These terms were computed in [9] by their direct method, which is based on the EulerMaclaurin summation formula and the Feynman-Kac formula, and they were checked by Gilkey's universal formulas. Our computations based on the method explained in the previous subsection also gives the same result.

The first term, whose integral up to a universal factor gives the volume, is given by

$$
a_{0}=\frac{a(t)^{3}}{2} .
$$

Since the latter appears as the leading term in the small time asymptotic expansion of the heat kernel it is related to Weyl's law, which reads the volume from the asymptotic distribution of the eigenvalues of $D^{2}$. The next term, which is related to the scalar curvature, has the expression

$$
a_{2}=\frac{1}{4} a(t)\left(a(t) a^{\prime \prime}(t)+a^{\prime}(t)^{2}-1\right) .
$$

The term after, whose integral is topological, is related to the Gauss-Bonnet term (cf. [9]) and is written as

$$
a_{4}=\frac{1}{120}\left(3 a^{(4)}(t) a(t)^{2}+3 a(t) a^{\prime \prime}(t)^{2}-5 a^{\prime \prime}(t)+9 a^{(3)}(t) a(t) a^{\prime}(t)-4 a^{\prime}(t)^{2} a^{\prime \prime}(t)\right) .
$$

The term $a_{6}$, which is the last term for which Gilkey's universal formulas are written, is given by

$$
\begin{aligned}
& a_{6}=\frac{1}{5040 a(t)^{2}}\left(9 a^{(6)}(t) a(t)^{4}-21 a^{(4)}(t) a(t)^{2}-3 a^{(3)}(t)^{2} a(t)^{3}-56 a(t)^{2} a^{\prime \prime}(t)^{3}+\right. \\
& 42 a(t) a^{\prime \prime}(t)^{2}+36 a^{(5)}(t) a(t)^{3} a^{\prime}(t)+6 a^{(4)}(t) a(t)^{3} a^{\prime \prime}(t)-42 a^{(4)}(t) a(t)^{2} a^{\prime}(t)^{2}+ \\
& 60 a^{(3)}(t) a(t) a^{\prime}(t)^{3}+21 a^{(3)}(t) a(t) a^{\prime}(t)+240 a(t) a^{\prime}(t)^{2} a^{\prime \prime}(t)^{2}-60 a^{\prime}(t)^{4} a^{\prime \prime}(t)- \\
& \left.21 a^{\prime}(t)^{2} a^{\prime \prime}(t)-252 a^{(3)}(t) a(t)^{2} a^{\prime}(t) a^{\prime \prime}(t)\right) .
\end{aligned}
$$

\subsection{The terms $a_{8}$ and $a_{10}$}

These terms were computed by Chamseddine and Connes in [9] using their direct method. In order to form a check on the final formulas, they have suggested to use the universal formulas of $[1,2,35]$ to calculate these terms and compare the results. As mentioned earlier, Gilkey's universal formulas were used in [9] to check the terms up to $a_{6}$, however, they are written in the literature only up to $a_{6}$ and become rather complicated even for this term.

In this subsection, we pursue the computation of the terms $a_{8}$ and $a_{10}$ in the expansion of the spectral action for Robertson-Walker metrics by continuing to employ pseudodifferential calculus, as presented in section 3.1, and check that the final formulas agree with the result in [9]. The final formulas for $a_{8}$ and $a_{10}$ are the following expressions:

$$
\begin{gathered}
a_{8}= \\
-\frac{1}{10080 a(t)^{4}}\left(-a^{(8)}(t) a(t)^{6}+3 a^{(6)}(t) a(t)^{4}+13 a^{(4)}(t)^{2} a(t)^{5}-24 a^{(3)}(t)^{2} a(t)^{3}-\right. \\
114 a(t)^{3} a^{\prime \prime}(t)^{4}+43 a(t)^{2} a^{\prime \prime}(t)^{3}-5 a^{(7)}(t) a(t)^{5} a^{\prime}(t)+2 a^{(6)}(t) a(t)^{5} a^{\prime \prime}(t)+9 a^{(6)}(t) a(t)^{4} a^{\prime}(t)^{2}+ \\
16 a^{(3)}(t) a^{(5)}(t) a(t)^{5}-24 a^{(5)}(t) a(t)^{3} a^{\prime}(t)^{3}-6 a^{(5)}(t) a(t)^{3} a^{\prime}(t)+69 a^{(4)}(t) a(t)^{4} a^{\prime \prime}(t)^{2}-
\end{gathered}
$$


$36 a^{(4)}(t) a(t)^{3} a^{\prime \prime}(t)+60 a^{(4)}(t) a(t)^{2} a^{\prime}(t)^{4}+15 a^{(4)}(t) a(t)^{2} a^{\prime}(t)^{2}+90 a^{(3)}(t)^{2} a(t)^{4} a^{\prime \prime}(t)-$ $216 a^{(3)}(t)^{2} a(t)^{3} a^{\prime}(t)^{2}-108 a^{(3)}(t) a(t) a^{\prime}(t)^{5}-27 a^{(3)}(t) a(t) a^{\prime}(t)^{3}+801 a(t)^{2} a^{\prime}(t)^{2} a^{\prime \prime}(t)^{3}-$ $588 a(t) a^{\prime}(t)^{4} a^{\prime \prime}(t)^{2}-87 a(t) a^{\prime}(t)^{2} a^{\prime \prime}(t)^{2}+108 a^{\prime}(t)^{6} a^{\prime \prime}(t)+27 a^{\prime}(t)^{4} a^{\prime \prime}(t)+$ $78 a^{(5)}(t) a(t)^{4} a^{\prime}(t) a^{\prime \prime}(t)+132 a^{(3)}(t) a^{(4)}(t) a(t)^{4} a^{\prime}(t)-312 a^{(4)}(t) a(t)^{3} a^{\prime}(t)^{2} a^{\prime \prime}(t)-$ $\left.819 a^{(3)}(t) a(t)^{3} a^{\prime}(t) a^{\prime \prime}(t)^{2}+768 a^{(3)}(t) a(t)^{2} a^{\prime}(t)^{3} a^{\prime \prime}(t)+102 a^{(3)}(t) a(t)^{2} a^{\prime}(t) a^{\prime \prime}(t)\right)$,

and

$$
a_{10}=
$$

$\frac{1}{665280 a(t)^{6}}\left(3 a^{(10)}(t) a(t)^{8}-222 a^{(5)}(t)^{2} a(t)^{7}-348 a^{(4)}(t) a^{(6)}(t) a(t)^{7}-147 a^{(3)}(t) a^{(7)}(t) a(t)^{7}-\right.$ $18 a^{\prime \prime}(t) a^{(8)}(t) a(t)^{7}+18 a^{\prime}(t) a^{(9)}(t) a(t)^{7}-482 a^{\prime \prime}(t) a^{(4)}(t)^{2} a(t)^{6}-331 a^{(3)}(t)^{2} a^{(4)}(t) a(t)^{6}-$ $1110 a^{\prime \prime}(t) a^{(3)}(t) a^{(5)}(t) a(t)^{6}-1556 a^{\prime}(t) a^{(4)}(t) a^{(5)}(t) a(t)^{6}-448 a^{\prime \prime}(t)^{2} a^{(6)}(t) a(t)^{6}-$ $1074 a^{\prime}(t) a^{(3)}(t) a^{(6)}(t) a(t)^{6}-476 a^{\prime}(t) a^{\prime \prime}(t) a^{(7)}(t) a(t)^{6}-43 a^{\prime}(t)^{2} a^{(8)}(t) a(t)^{6}-$ $11 a^{(8)}(t) a(t)^{6}+8943 a^{\prime}(t) a^{(3)}(t)^{3} a(t)^{5}+21846 a^{\prime \prime}(t)^{2} a^{(3)}(t)^{2} a(t)^{5}+4092 a^{\prime}(t)^{2} a^{(4)}(t)^{2} a(t)^{5}+$ $396 a^{(4)}(t)^{2} a(t)^{5}+10560 a^{\prime \prime}(t)^{3} a^{(4)}(t) a(t)^{5}+39402 a^{\prime}(t) a^{\prime \prime}(t) a^{(3)}(t) a^{(4)}(t) a(t)^{5}+$ $11352 a^{\prime}(t) a^{\prime \prime}(t)^{2} a^{(5)}(t) a(t)^{5}+6336 a^{\prime}(t)^{2} a^{(3)}(t) a^{(5)}(t) a(t)^{5}+594 a^{(3)}(t) a^{(5)}(t) a(t)^{5}+$ $2904 a^{\prime}(t)^{2} a^{\prime \prime}(t) a^{(6)}(t) a(t)^{5}+264 a^{\prime \prime}(t) a^{(6)}(t) a(t)^{5}+165 a^{\prime}(t)^{3} a^{(7)}(t) a(t)^{5}+$ $33 a^{\prime}(t) a^{(7)}(t) a(t)^{5}-10338 a^{\prime \prime}(t)^{5} a(t)^{4}-95919 a^{\prime}(t)^{2} a^{\prime \prime}(t) a^{(3)}(t)^{2} a(t)^{4}-$ $3729 a^{\prime \prime}(t) a^{(3)}(t)^{2} a(t)^{4}-117600 a^{\prime}(t) a^{\prime \prime}(t)^{3} a^{(3)}(t) a(t)^{4}-68664 a^{\prime}(t)^{2} a^{\prime \prime}(t)^{2} a^{(4)}(t) a(t)^{4}-$ $2772 a^{\prime \prime}(t)^{2} a^{(4)}(t) a(t)^{4}-23976 a^{\prime}(t)^{3} a^{(3)}(t) a^{(4)}(t) a(t)^{4}-2640 a^{\prime}(t) a^{(3)}(t) a^{(4)}(t) a(t)^{4}-$ $12762 a^{\prime}(t)^{3} a^{\prime \prime}(t) a^{(5)}(t) a(t)^{4}-1386 a^{\prime}(t) a^{\prime \prime}(t) a^{(5)}(t) a(t)^{4}-651 a^{\prime}(t)^{4} a^{(6)}(t) a(t)^{4}-$ $132 a^{\prime}(t)^{2} a^{(6)}(t) a(t)^{4}+111378 a^{\prime}(t)^{2} a^{\prime \prime}(t)^{4} a(t)^{3}+2354 a^{\prime \prime}(t)^{4} a(t)^{3}+31344 a^{\prime}(t)^{4} a^{(3)}(t)^{2} a(t)^{3}+$ $3729 a^{\prime}(t)^{2} a^{(3)}(t)^{2} a(t)^{3}+236706 a^{\prime}(t)^{3} a^{\prime \prime}(t)^{2} a^{(3)}(t) a(t)^{3}+13926 a^{\prime}(t) a^{\prime \prime}(t)^{2} a^{(3)}(t) a(t)^{3}+$ $43320 a^{\prime}(t)^{4} a^{\prime \prime}(t) a^{(4)}(t) a(t)^{3}+5214 a^{\prime}(t)^{2} a^{\prime \prime}(t) a^{(4)}(t) a(t)^{3}+2238 a^{\prime}(t)^{5} a^{(5)}(t) a(t)^{3}+$ $462 a^{\prime}(t)^{3} a^{(5)}(t) a(t)^{3}-162162 a^{\prime}(t)^{4} a^{\prime \prime}(t)^{3} a(t)^{2}-11880 a^{\prime}(t)^{2} a^{\prime \prime}(t)^{3} a(t)^{2}-$ $103884 a^{\prime}(t)^{5} a^{\prime \prime}(t) a^{(3)}(t) a(t)^{2}-13332 a^{\prime}(t)^{3} a^{\prime \prime}(t) a^{(3)}(t) a(t)^{2}-6138 a^{\prime}(t)^{6} a^{(4)}(t) a(t)^{2}-$ $1287 a^{\prime}(t)^{4} a^{(4)}(t) a(t)^{2}+76440 a^{\prime}(t)^{6} a^{\prime \prime}(t)^{2} a(t)+10428 a^{\prime}(t)^{4} a^{\prime \prime}(t)^{2} a(t)+$ $\left.11700 a^{\prime}(t)^{7} a^{(3)}(t) a(t)+2475 a^{\prime}(t)^{5} a^{(3)}(t) a(t)-11700 a^{\prime}(t)^{8} a^{\prime \prime}(t)-2475 a^{\prime}(t)^{6} a^{\prime \prime}(t)\right)$.

\section{Computation of the term $a_{12}$ in the expansion of the spectral action}

We pursue the computation of the term $a_{12}$ in the expansion of the spectral action for Robertson-Walker metrics by employing pseudodifferential calculus to find the term $r_{12}$ for the parametrix of $\lambda-D^{2}$, which is homogeneous of order -14 , and by performing the appropriate integrations. Since there is no universal formula in the literature for this term, we have performed two heavy computations, one in Hopf coordinates and the other in spherical coordinates, to form a check on the validity of the outcome of our calculations. Another efficient way of computing the term $a_{12}$ is to use the direct method of [9].

\subsection{The result of the computation in Hopf coordinates}

Continuing the recursive procedure commenced in the previous section and exploiting computer assistance, while the calculation becomes significantly heavier for the term $a_{12}$, we find the following expression:

$$
a_{12}=
$$




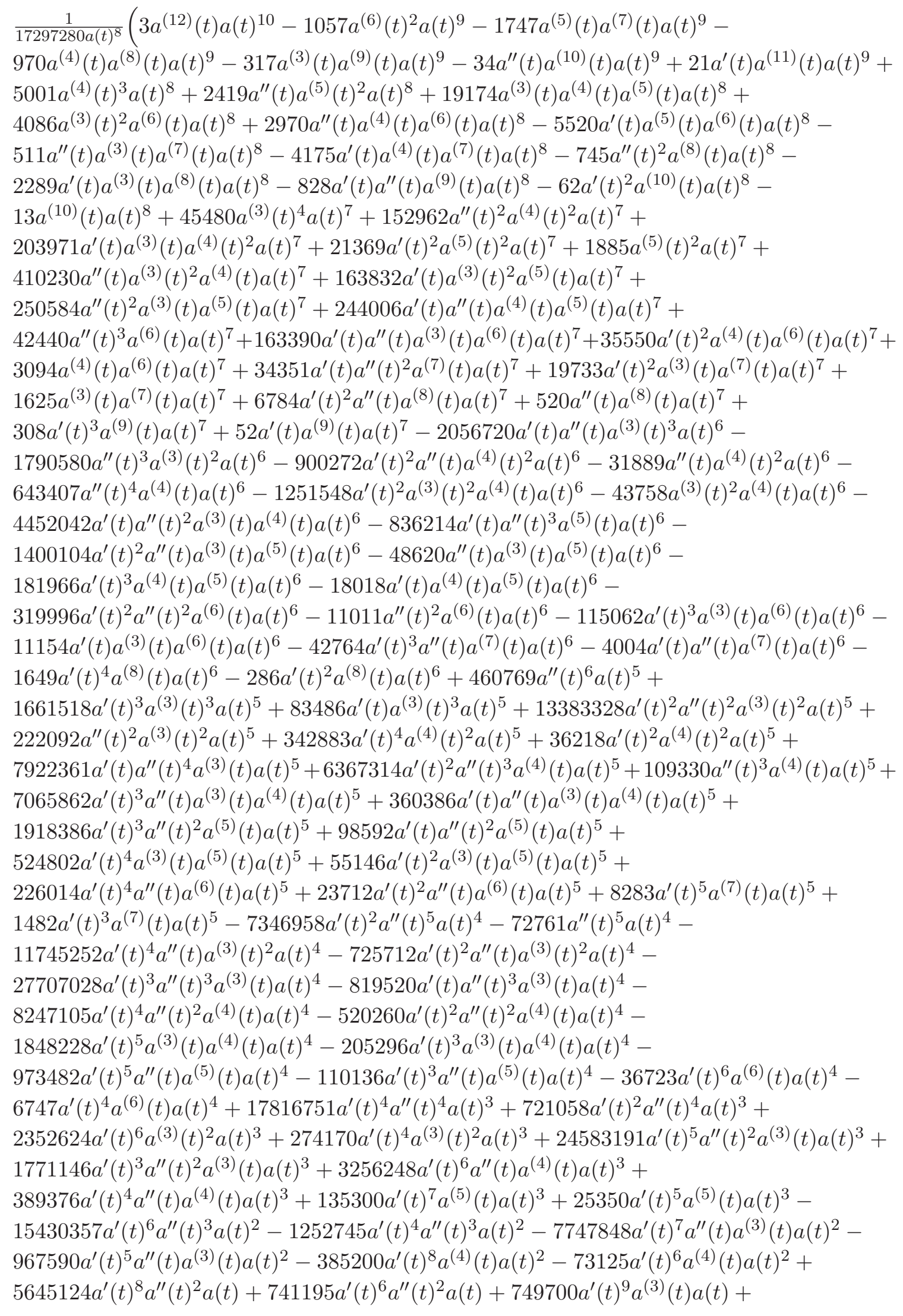


$\left.\left.143325 a^{\prime}(t)^{7} a^{(3)}(t) a(t)-749700 a^{\prime}(t)^{10} a^{\prime \prime}(t)-143325 a^{\prime}(t)^{8} a^{\prime \prime}(t)\right)\right)$.

\subsection{Agreement of the result with computations in spherical coordinates}

Taking a similar route as in section 2, we explicitly write the Dirac operator for the Roberson-Walker metric in spherical coordinates

$$
d s^{2}=d t^{2}+a^{2}(t)\left(d \chi^{2}+\sin ^{2}(\chi)\left(d \theta^{2}+\sin ^{2}(\theta) d \varphi^{2}\right)\right) .
$$

Using the computations carried out in [9] with the orthonormal coframe

$$
d t, \quad a(t) d \chi, \quad a(t) \sin \chi d \theta, \quad a(t) \sin \chi \sin \theta d \varphi,
$$

the corresponding matrix of connection 1-forms for the Levi-Civita connection is written as

$$
\left(\begin{array}{cccc}
0 & -a^{\prime}(t) d \chi & -a^{\prime}(t) \sin (\chi) d \theta & -a^{\prime}(t) \sin (\chi) \sin (\theta) d \varphi \\
a^{\prime}(t) d \chi & 0 & -\cos (\chi) d \theta & -\cos (\chi) \sin (\theta) d \varphi \\
a^{\prime}(t) \sin (\chi) d \theta & \cos (\chi) d \theta & 0 & -\cos (\theta) d \varphi \\
a^{\prime}(t) \sin (\chi) \sin (\theta) d \varphi & \cos (\chi) \sin (\theta) d \varphi & \cos (\theta) d \varphi & 0
\end{array}\right)
$$

Lifting to the spin bundle by means of the Lie algebra isomorphism $\mu: \mathfrak{s o}(4) \rightarrow$ $\mathfrak{s p i n}(4)$ and writing the formula for the Dirac operator yield the following expression for this operator expressed in spherical coordiantes:

$$
\begin{aligned}
D= & \gamma^{1} \frac{\partial}{\partial t}+\gamma^{2} \frac{1}{a} \frac{\partial}{\partial \chi}+\gamma^{3} \frac{1}{a \sin \chi} \frac{\partial}{\partial \theta}+\gamma^{4} \frac{1}{a \sin \chi \sin \theta} \frac{\partial}{\partial \varphi} \\
& +\frac{3 a^{\prime}}{2 a} \gamma^{1}+\frac{\cot (\chi)}{a} \gamma^{2}+\frac{\cot (\theta)}{2 a \sin (\chi)} \gamma^{3}
\end{aligned}
$$

Thus the pseudodifferential symbol of $D$ is given by

$$
\begin{aligned}
\sigma_{D}(x, \xi)= & i \gamma^{1} \xi_{1}+\frac{i}{a} \gamma^{2} \xi_{2}+\frac{i}{a \sin (\chi)} \gamma^{3} \xi_{3}+\frac{i}{a \sin (\chi) \sin (\theta)} \gamma^{4} \xi_{4} \\
& +\frac{3 a^{\prime}}{2 a} \gamma^{1}+\frac{\cot (\chi)}{a} \gamma^{2}+\frac{\cot (\theta)}{2 a \sin (\chi)} \gamma^{3} .
\end{aligned}
$$

Accordingly, the symbol of $D^{2}$ is the sum $p_{2}^{\prime}+p_{1}^{\prime}+p_{0}^{\prime}$ of three homogeneous components

$$
\begin{aligned}
p_{2}^{\prime}= & \xi_{1}^{2}+\frac{1}{a(t)^{2}} \xi_{2}^{2}+\frac{1}{a(t)^{2} \sin ^{2}(\chi)} \xi_{3}^{2}+\frac{1}{a(t)^{2} \sin ^{2}(\theta) \sin ^{2}(\chi)} \xi_{4}^{2} \\
p_{1}^{\prime}= & -\frac{3 i a^{\prime}(t)}{a(t)} \xi_{1}-\frac{i}{a(t)^{2}}\left(\gamma^{12} a^{\prime}(t)+2 \cot (\chi)\right) \xi_{2} \\
& -\frac{i}{a(t)^{2}}\left(\gamma^{13} \csc (\chi) a^{\prime}(t)+\cot (\theta) \csc ^{2}(\chi)+\gamma^{23} \cot (\chi) \csc (\chi)\right) \xi_{3} \\
& -\frac{i}{a(t)^{2}}\left(\csc (\theta) \csc (\chi) a^{\prime}(t) \gamma^{14}+\cot (\theta) \csc (\theta) \csc ^{2}(\chi) \gamma^{34}\right. \\
& \left.+\csc (\theta) \cot (\chi) \csc (\chi) \gamma^{24}\right) \xi_{4}, \\
p_{0}^{\prime}= & \frac{1}{8 a(t)^{2}}\left(-12 a(t) a^{\prime \prime}(t)-6 a^{\prime}(t)^{2}+3 \csc ^{2}(\theta) \csc ^{2}(\chi)-\cot ^{2}(\theta) \csc ^{2}(\chi)+\right.
\end{aligned}
$$




$$
\begin{aligned}
& \left.+4 i \cot (\theta) \cot (\chi) \csc (\chi)-4 i \cot (\theta) \cot (\chi) \csc (\chi)-4 \cot ^{2}(\chi)+5 \csc ^{2}(\chi)+4\right) \\
& -\frac{\left(\cot (\theta) \csc (\chi) a^{\prime}(t)\right)}{2 a(t)^{2}} \gamma^{13}-\frac{\left(\cot (\chi) a^{\prime}(t)\right)}{a(t)^{2}} \gamma^{12}-\frac{(\cot (\theta) \cot (\chi) \csc (\chi))}{2 a(t)^{2}} \gamma^{23}
\end{aligned}
$$

We have performed the computation of the heat coefficients up to the term $a_{12}$ using the latter symbols and have checked the agreement of the result with the computations in Hopf coordinates, presented in the previous subsections. This is in particular of great importance for the term $a_{12}$, since it ensures the validity of our computations performed in two different coordinates.

\subsection{Agreement with the full expansion for the round metric}

We first recall the full expansion for the spectral action for the round metric, namely the case $a(t)=\sin (t)$, worked out in [9]. Then we show that the term $a_{12}$ presented in section 4.1 reduces correctly to the round case.

The method devised in [9] has wide applicability in the spectral action computations since it can be used for the cases when the eigenvalues of the square of the Dirac operator have a polynomial expression while their multiplicities are also given by polynomials. In the case of the round metric on $\mathbb{S}^{4}$, after remarkable computations based on the EulerMaclaurin formula, this method leads to the following expression with control over the remainder term [9]:

$$
\begin{aligned}
\frac{3}{4} \operatorname{Trace}\left(f\left(t D^{2}\right)\right)= & \int_{0}^{\infty} f\left(t x^{2}\right)\left(x^{3}-x\right) d x+\frac{11 f(0)}{120}-\frac{31 f^{\prime}(0) t}{2520}+\frac{41 f^{\prime \prime}(0) t^{2}}{10080} \\
& -\frac{31 f^{(3)}(0) t^{3}}{15840}+\frac{10331 f^{(4)}(0) t^{4}}{8648640}-\frac{3421 f^{(5)}(0) t^{5}}{3931200}+\cdots+R_{m} .
\end{aligned}
$$

This implies that the term $a_{12}$ in the expansion of the spectral action for the round metric is equal to $\frac{10331}{6486480}$. To check our calculations against this result, we find that for $a(t)=\sin (t)$ the expression for $a_{12}(t)$ reduces to $\frac{10331 \sin ^{3}(t)}{8648640}$, and hence

$$
a_{12}=\int_{0}^{\pi} a_{12}\left(\mathbb{S}^{4}\right) d t=\frac{4}{3} \frac{10331}{8648640}=\frac{10331}{6486480},
$$

which is in complete agreement with the result in [9], mentioned above.

\section{Chameseddine-Connes' conjecture}

In this section we prove a conjecture of Chamseddine and Connes from [9]. More precisely, we show that the term $a_{2 n}$ in the asymptotic expansion of the spectral action for RobertsonWalker metrics is, up to multiplication by $a(t)^{3-2 n}$, of the form $Q_{2 n}\left(a, a^{\prime}, \ldots, a^{(2 n)}\right)$, where $Q_{2 n}$ is a polynomial with rational coefficients.

\subsection{Proof of rationality of the coefficients in the expressions for $a_{2 n}$}

A crucial point that enables us to furnish the proof of our main theorem, namely the proof of the conjecture mentioned above, is the independence of the integral kernel of the heat 
operator of the Dirac operator of the Robertson-Walker metric from the variables $\phi_{1}, \phi_{2}, \eta$. Note that since the symbol and the metric are independent of $\phi_{1}, \phi_{2}$, the computations involved in the symbol calculus clearly imply the independence of the terms $e_{n}$ from these variables. However, the independence of $e_{n}$ from $\eta$ is not evident, which is proved as follows.

Lemma 1. The heat kernel $k(t, x, x)$ for the Robertson-Walker metric is independent of $\phi_{1}, \phi_{2}, \eta$.

Proof. The round metric on $\mathbb{S}^{3}$ is the bi-invariant metric on $\mathrm{SU}(2)$ induced from the Killing form of its Lie algebra $\mathfrak{s u}(2)$. The corresponding Levi-Civita connection restricted to the left invariant vector fields is given by $\frac{1}{2}[X, Y]$, and to the right invariant vector fields by $\frac{-1}{2}[X, Y]$. Since the Killing form is ad-invariant, we have

$$
\langle[X, Y], Z\rangle+\langle Y,[X, Z]\rangle=0, \quad X, Y, Z \in \mathfrak{s u}(2),
$$

which implies that in terms of the connection on left (right) invariant vector fields $X, Y, Z$, it can be written as

$$
\left\langle\nabla_{Y} X, Z\right\rangle+\left\langle Y, \nabla_{Z} X\right\rangle=0 .
$$

Considering the fact that $\nabla X: \mathfrak{X}(M) \rightarrow \mathfrak{X}(M)$ is an endomorphism of the tangent bundle, the latter identity holds for any $Y, Z \in \mathfrak{X}(M)$. Therefore, the equation (5.1) is the Killing equation and shows that any left and right invariant vector field on $\mathrm{SU}(2)$ is a Killing vector field.

By direct computation in Hopf coordinates, we find the following vector fields which respectively form bases for left and right invariant vector fields on $\mathrm{SU}(2)$ :

$$
\begin{aligned}
X_{1}^{L} & =\frac{\partial}{\partial \phi_{1}}+\frac{\partial}{\partial \phi_{2}}, \\
X_{2}^{L} & =\sin \left(\phi_{1}+\phi_{2}\right) \frac{\partial}{\partial \eta}+\cot (\eta) \cos \left(\phi_{1}+\phi_{2}\right) \frac{\partial}{\partial \phi_{1}}-\tan (\eta) \cos \left(\phi_{1}+\phi_{2}\right) \frac{\partial}{\partial \phi_{2}} \\
X_{3}^{L} & =\cos \left(\phi_{1}+\phi_{2}\right) \frac{\partial}{\partial \eta}-\cot (\eta) \sin \left(\phi_{1}+\phi_{2}\right) \frac{\partial}{\partial \phi_{1}}+\tan (\eta) \sin \left(\phi_{1}+\phi_{2}\right) \frac{\partial}{\partial \phi_{2}} \\
X_{1}^{R} & =-\frac{\partial}{\partial \phi_{1}}+\frac{\partial}{\partial \phi_{2}} \\
X_{2}^{R} & =-\sin \left(\phi_{1}-\phi_{2}\right) \frac{\partial}{\partial \eta}-\cot (\eta) \cos \left(\phi_{1}-\phi_{2}\right) \frac{\partial}{\partial \phi_{1}}-\tan (\eta) \cos \left(\phi_{1}-\phi_{2}\right) \frac{\partial}{\partial \phi_{2}} \\
X_{3}^{R} & =\cos \left(\phi_{1}-\phi_{2}\right) \frac{\partial}{\partial \eta}-\cot (\eta) \sin \left(\phi_{1}-\phi_{2}\right) \frac{\partial}{\partial \phi_{1}}-\tan (\eta) \sin \left(\phi_{1}-\phi_{2}\right) \frac{\partial}{\partial \phi_{2}}
\end{aligned}
$$

One can check that these vector fields are indeed Killing vector fields for the RobertsonWalker metrics on the four dimensional space. Thus, for any isometry invariant function $f$ we have:

$$
\begin{aligned}
\frac{\partial}{\partial \phi_{1}} f & =\frac{1}{2}\left(X_{1}^{L}-X_{1}^{R}\right) f=0, \\
\frac{\partial}{\partial \phi_{2}} f & =\frac{1}{2}\left(X_{1}^{L}+X_{1}^{R}\right) f=0,
\end{aligned}
$$




$$
\frac{\partial}{\partial \eta} f=\left(\sin \left(\phi_{1}+\phi_{2}\right) X_{2}^{L}+\cos \left(\phi_{1}+\phi_{2}\right) X_{3}^{L}\right) f=0 .
$$

In particular, the heat kernel restricted to the diagonal, $k(t, x, x)$, is independent of $\phi_{1}, \phi_{2}, \eta$, and so are the coefficient functions $e_{n}$ in its asymptotic expansion.

We stress that although $e_{n}(x)$ is independent of $\eta, \phi_{1}, \phi_{2}$, its components denoted by $e_{n, j, \alpha}$ in the proof of the following theorem are not necessarily independent of these variables.

Theorem 1. The term $a_{2 n}$ in the expansion of the spectral action for the Robertson-Walker metric with cosmic scale factor $a(t)$ is of the form

$$
\frac{1}{a(t)^{2 n-3}} Q_{2 n}\left(a(t), a^{\prime}(t), \ldots, a^{(2 n)}(t)\right)
$$

where $Q_{2 n}$ is a polynomial with rational coefficients.

Proof. Using (3.5) we can write

$$
e_{n}=\sum_{\substack{2 j-2-|\alpha|=n \\ n / 2+1 \leq j \leq 2 n+1}} c_{\alpha} e_{n, j, \alpha},
$$

where

$$
e_{n, j, \alpha}=\frac{1}{(j-1) !} r_{n, j, \alpha} a(t)^{\alpha_{2}+\alpha_{3}+\alpha_{4}} \sin (\eta)^{\alpha_{3}} \cos (\eta)^{\alpha_{4}} .
$$

The recursive equation (3.6) implies that

$$
\begin{aligned}
& e_{n, j, \alpha}= \\
& \frac{1}{(j-1) a(t)}\left(\left(\gamma^{14} a^{\prime}(t)-\tan (\eta) \gamma^{24}\right) e_{n-1, j-1, \alpha-\mathbf{e}_{4}}+\left(\gamma^{13} a^{\prime}(t)+\cot (\eta) \gamma^{23}\right) e_{n-1, j-1, \alpha-\mathbf{e}_{3}}+\right. \\
& \left(\gamma^{12} a^{\prime}(t)+1\left(\left(2 \alpha_{4}-1\right) \tan (\eta)+\left(1-2 \alpha_{3}\right) \cot (\eta)\right)\right) e_{n-1, j-1, \alpha-\mathbf{e}_{2}}+4 a^{\prime}(t) e_{n-1, j-2, \alpha-\mathbf{e}_{1}-2 \mathbf{e}_{2}}+ \\
& 4 a^{\prime}(t) e_{n-1, j-2, \alpha-\mathbf{e}_{1}-2 \mathbf{e}_{3}}+4 a^{\prime}(t) e_{n-1, j-2, \alpha-\mathbf{e}_{1}-2 \mathbf{e}_{4}}+\left(-2 \alpha_{2}-2 \alpha_{3}-2 \alpha_{4}+\right. \\
& \text { 3) } a^{\prime}(t) e_{n-1, j-1, \alpha-\mathbf{e}_{1}}+2 a(t) \frac{\partial}{\partial t} e_{n-1, j-1, \alpha-\mathbf{e}_{1}}-4 \tan (\eta) e_{n-1, j-2, \alpha-\mathbf{e}_{2}-2 \mathbf{e}_{4}}+ \\
& \left.4 \cot (\eta) e_{n-1, j-2, \alpha-\mathbf{e}_{2}-2 \mathbf{e}_{3}}+2 \frac{\partial}{\partial \eta} e_{n-1, j-1, \alpha-\mathbf{e}_{2}}\right) \\
& +\frac{1}{(j-1) a(t)^{2}}\left(a(t)^{2} \frac{\partial^{2}}{\partial t^{2}} e_{n-2, j-1, \alpha}+4 a^{\prime}(t) a(t) \frac{\partial}{\partial t} e_{n-2, j-2, \alpha-2 \mathbf{e}_{2}}+4 a^{\prime}(t) a(t) \frac{\partial}{\partial t} e_{n-2, j-2, \alpha-2 \mathbf{e}_{3}}+\right. \\
& 4 a^{\prime}(t) a(t) \frac{\partial}{\partial t} e_{n-2, j-2, \alpha-2 \mathbf{e}_{4}}+\left(-2 \alpha_{2}-2 \alpha_{3}-2 \alpha_{4}+3\right) a^{\prime}(t) a(t) \frac{\partial}{\partial t} e_{n-2, j-1, \alpha}+ \\
& 4 a^{\prime}(t)^{2} e_{n-2, j-3, \alpha-4 \mathbf{e}_{2}}+8 a^{\prime}(t)^{2} e_{n-2, j-3, \alpha-2 \mathbf{e}_{2}-2 \mathbf{e}_{3}}+8 a^{\prime}(t)^{2} e_{n-2, j-3, \alpha-2 \mathbf{e}_{2}-2 \mathbf{e}_{4}}+ \\
& 4 \cot (\eta) \frac{\partial}{\partial \eta} e_{n-2, j-2, \alpha-2 \mathbf{e}_{3}}-4 \tan (\eta) \frac{\partial}{\partial \eta} e_{n-2, j-2, \alpha-2 \mathbf{e}_{4}}+\frac{\partial^{2}}{\partial \eta^{2}} e_{n-2, j-1, \alpha}+\left(2 \cot (\eta) \gamma^{12} a^{\prime}(t)+\right. \\
& \left.\left(-4\left(\alpha_{2}+\alpha_{3}+\alpha_{4}-2\right) a^{\prime}(t)^{2}+4\left(-\left(\alpha_{3}-1\right) \csc ^{2}(\eta)+\alpha_{3}+\alpha_{4}-2\right)+2 a(t) a^{\prime \prime}(t)\right)\right) e_{n-2, j-2, \alpha-2 \mathbf{e}_{3}}+ \\
& \left(\left(\cot (\eta)\left(1-2 \alpha_{3}\right)+\left(2 \alpha_{4}-1\right) \tan (\eta)\right)+\gamma^{12} a^{\prime}(t)\right) \frac{\partial}{\partial \eta} e_{n-2, j-1, \alpha}+\left(\left(-4\left(\alpha_{2}+\alpha_{3}+\alpha_{4}-2\right) a^{\prime}(t)^{2}+\right.\right. \\
& \left.\left.4\left(-\left(\alpha_{4}-1\right) \sec ^{2}(\eta)+\alpha_{3}+\alpha_{4}-2\right)+2 a(t) a^{\prime \prime}(t)\right)-2 \gamma^{12} \tan (\eta) a^{\prime}(t)\right) e_{n-2, j-2, \alpha-2 \mathbf{e}_{4}}+8\left(a^{\prime}(t)^{2}-\right. \\
& \text { 1) } e_{n-2, j-3, \alpha-2 \mathbf{e}_{4}}+4\left(\cot ^{2}(\eta)+a^{\prime}(t)^{2}\right) e_{n-2, j-3, \alpha-4 \mathbf{e}_{3}}+4\left(\tan ^{2}(\eta)+a^{\prime}(t)^{2}\right) e_{n-2, j-3, \alpha-4 \mathbf{e}_{4}}+ \\
& \left(2 a(t) a^{\prime \prime}(t)-4\left(\alpha_{2}+\alpha_{3}+\alpha_{4}-2\right) a^{\prime}(t)^{2}\right) e_{n-2, j-2, \alpha-2 \mathbf{e}_{2}}+\left(\frac { 1 } { 2 } \left(\cot (\eta)\left(1-2 \alpha_{3}\right)+\left(2 \alpha_{4}-\right.\right.\right. \\
& \text { 1) } \tan (\eta)) \gamma^{12} a^{\prime}(t)+\frac{1}{4}\left(\left(4 \alpha_{3}^{2}-1\right) \csc ^{2}(\eta)-4\left(\alpha_{3}+\alpha_{4}-1\right)^{2}+\left(2 \alpha_{2}+2 \alpha_{3}+2 \alpha_{4}-3\right)\left(2 \alpha_{2}+\right.\right. \\
& \left.\left.\left.\left.2 \alpha_{3}+2 \alpha_{4}-1\right) a^{\prime}(t)^{2}+\sec ^{2}(\eta)\left(4 \alpha_{4}^{2}-1\right)-2\left(2 \alpha_{2}+2 \alpha_{3}+2 \alpha_{4}-3\right) a(t) a^{\prime \prime}(t)\right)\right) e_{n-2, j-1, \alpha}\right) \text {. }
\end{aligned}
$$


The functions associated with the initial indices are:

$$
\begin{aligned}
& e_{0,1,0,0,0,0}=1, \quad e_{1,2,1,0,0,0}=\frac{3 i a^{\prime}(t)}{a(t)}, \quad e_{1,3,1,2,0,0}=\frac{2 i a^{\prime}(t)}{a(t)} \\
& e_{1,3,1,0,2,0}=\frac{2 i a^{\prime}(t)}{a(t)}, \quad e_{1,3,1,0,0,2}=\frac{2 i a^{\prime}(t)}{a(t)}, \quad e_{1,3,0,1,0,2}=-\frac{(2 i) \tan (\eta)}{a(t)} \\
& e_{1,3,0,1,2,0}=\frac{(2 i) \cot (\eta)}{a(t)}, \quad e_{1,2,0,0,1,0}=\frac{i \gamma^{13} a^{\prime}(t)}{a(t)}+\frac{i \gamma^{23} \cot (\eta)}{a(t)} \\
& e_{1,2,0,0,0,1}=\frac{i \gamma^{14} a^{\prime}(t)}{a(t)}-\frac{i \gamma^{24} \tan (\eta)}{a(t)}, \quad e_{1,2,0,1,0,0}=\frac{2 i \cot (2 \eta)}{a(t)}+\frac{i \gamma^{12} a^{\prime}(t)}{a(t)} .
\end{aligned}
$$

It is then apparent that $e_{0}$ and $e_{1}$ are, respectively, a polynomial in $a(t)$, and a polynomial in $a(t)$ and $a^{\prime}(t)$, divided by some powers of $a(t)$. Thus, it follows from the above recursive formula that all $e_{n, j, \alpha}$ are of this form. Accordingly, we have

$$
e_{n}=\frac{P_{n}}{a(t)^{d_{n}}},
$$

where $P_{n}$ is a polynomial in $a(t)$ and its derivatives with matrix coefficients. Writing $e_{n, j, \alpha}=P_{n, j, \alpha} / a(t)^{d_{n}}$, we obtain $d_{n}=\max \left\{d_{n-1}+1, d_{n-2}+2\right\}$. Starting with $d_{0}=0$, $d_{1}=-1$, and following to obtain $d_{n}=n$, we conclude that

$$
e_{n, j, \alpha}=\frac{1}{a^{n}(t)} P_{n, j, \alpha}\left(a(t), \ldots, a^{(n)}(t)\right)
$$

where $P_{n, j, \alpha}$ is a polynomial whose coefficients are matrices with entries in the algebra generated by $\sin (\eta), \csc (\eta), \cos (\eta), \sec (\eta)$ and rational numbers.

In the calculation of the even terms $a_{2 n}$, only even $\alpha_{k}$ have contributions in the summation (5.2). This implies that the corresponding $c_{\alpha}$ is a rational multiple of $\pi^{2}$ and $P_{2 n}$ is a polynomial with rational matrix coefficients, which is independent of variables $\eta, \phi_{1}, \phi_{2}$ by Lemma 1 . Hence

$$
a_{2 n}=\frac{1}{16 \pi^{4}} \int_{\mathbb{S}_{a}^{3}} \operatorname{tr}\left(e_{2 n}\right) d v o l_{g}=\frac{2 \pi^{2} a(t)^{3}}{16 \pi^{4}} \operatorname{tr}\left(\frac{P_{2 n}}{a(t)^{2 n}}\right)=\frac{Q_{2 n}}{a(t)^{n-3}},
$$

where $Q_{2 n}$ is a polynomial in $a(t), a^{\prime}(t), \ldots, a^{(2 n)}(t)$ with rational coefficients.

The polynomials $P_{n, j, \alpha}$ also satisfy recursive relations that illuminate interesting features about their structure.

Proposition 2. Each $P_{n, j, \alpha}$ is a finite sum of the form

$$
\sum c_{k} a(t)^{k_{0}} a^{\prime}(t)^{k_{1}} \cdots a^{(n)}(t)^{k_{n}}
$$

where each $c_{k}$ is a matrix of functions that are independent from the variable $t$, and $\sum_{j=0}^{n} k_{j}=\sum_{j=0}^{n} j k_{j}=l$, for some $0 \leq l \leq n$. 
Proof. This follows from an algebraically lengthy recursive formula for $P_{n, j, \alpha}$ which stems from the equation (3.6), similar to the recursive formula for $e_{n, j, \alpha}$ in the proof of Theorem 1. In addition, one needs to find the following initial cases:

$$
\begin{aligned}
& P_{0,1,0,0,0,0}=I, \quad P_{1,2,1,0,0,0}=3 i a^{\prime}(t), \quad P_{1,2,0,0,1,0}=i \gamma^{13} a^{\prime}(t)+i \gamma^{23} \cot (\eta), \\
& P_{1,2,0,0,0,1}=i \gamma^{14} a^{\prime}(t)-i \gamma^{24} \tan (\eta), \quad P_{1,2,0,1,0,0}=2 i \cot (2 \eta)+i \gamma^{12} a^{\prime}(t), \\
& P_{1,3,0,1,0,2}=-2 i \tan (\eta), \quad P_{1,3,0,1,2,0}=2 i \cot (\eta), \quad P_{1,3,1,2,0,0}=2 i a^{\prime}(t), \\
& P_{1,3,1,0,2,0}=2 i a^{\prime}(t), \quad P_{1,3,1,0,0,2}=2 i a^{\prime}(t) .
\end{aligned}
$$

\subsection{A recursive formula for the coefficient of the highest order term in $a_{2 n}$}

The highest derivative of the cosmic scale factor $a(t)$ in the expression for $a_{n}$ is seen in the term $a(t)^{n-1} a^{(n)}(t)$, which has a rational coefficient based on Theorem 1. Let us denote the coefficient of $a(t)^{n-1} a^{(n)}(t)$ in $a_{n}$ by $h_{n}$. Since the coefficients $h_{n}$ are limited to satisfy the recursive relations derived in the proof of the following proposition, one can find the following closed formula for these coefficients.

Proposition 3. The coefficient $h_{n}$ of $a(t)^{n-1} a^{(n)}(t)$ in $a_{n}$ is equal to

$$
\begin{aligned}
& \sum_{[n / 2]+1 \leq j \leq 2 n+1} \Gamma\left(\frac{2 k+1}{2}\right) H_{n, j, 2 k}, \\
& {[n / 2]+1 \leq j \leq 2 n+1} \\
& 0 \leq k \leq j-n / 2-1
\end{aligned}
$$

where, starting from

$$
\begin{array}{ll}
H_{1,2,1}=H_{1,3,1}=\frac{3 i}{2 \sqrt{\pi}}, & H_{2,4,2}=-\frac{1}{\sqrt{\pi}}, \\
H_{2,3,0}=H_{2,2,0}=\frac{3}{4 \sqrt{\pi}}, & H_{2,3,2}=-\frac{3}{2 \sqrt{\pi}},
\end{array}
$$

the quantities $H_{n, j, \alpha}$ are computed recursively by

$$
H_{n, j, \alpha}=\frac{1}{j-1}\left(H_{n-2, j-1, \alpha}+2 i H_{n-1, j-1, \alpha-1}\right) .
$$

Proof. It follows from Proposition 2 that the highest derivative of $a(t)$ in $a_{n}$ appears in the term $a(t)^{n-1} a^{(n)}(t)$. By a careful analysis of the equation (5.3) we find that only the terms

$$
\frac{1}{j-1}\left(a(t)^{2} \frac{\partial^{2}}{\partial t^{2}} P_{n-2, j-1, \alpha}+2 i a(t) \frac{\partial}{\partial t} P_{n-1, j-1, \alpha-\mathbf{e}_{1}}\right)
$$

contribute to its recursive formula. Denoting the corresponding monomial in $P_{n, j, \alpha}$ by $H_{n, j, \alpha} a(t)^{n-1} a^{(n)}(t)$ and substituting it into the above formula we obtain the equation

$$
H_{n, j, \alpha}=\frac{1}{j-1}\left(H_{n-2, j-1, \alpha}+2 i H_{n-1, j-1, \alpha-\mathbf{e}_{1}}\right),
$$


for any $n>2$. Denoting

$$
H_{n, j, \alpha_{1}}=\sum \prod_{k=2}^{4} \Gamma\left(\frac{\alpha_{k}+1}{2}\right) \frac{(-1)^{\alpha_{k}}+1}{2} \operatorname{tr}\left(\frac{1}{(2 \pi)^{2}} \int_{0}^{\pi / 2} H_{n, j, \alpha_{1}, \alpha_{2}, \alpha_{3}, \alpha_{4}} d \eta\right),
$$

the recursive formula converts to

$$
H_{n, j, \alpha}=\frac{1}{j-1}\left(H_{n-2, j-1, \alpha}+2 i H_{n-1, j-1, \alpha-1}\right) .
$$

Thus, the coefficient of $a(t)^{n-1} a^{(n)}(t)$ in $a_{n}$ is given by the above expression.

Using the above proposition we find that:

$$
\begin{aligned}
h_{2} & =\frac{1}{4}, \quad h_{4}=\frac{1}{40}, \quad h_{6}=\frac{1}{560}, \quad h_{8}=\frac{1}{10080}, \quad h_{10}=\frac{1}{221760}, \\
h_{12} & =\frac{1}{5765760}, \quad h_{14}=\frac{1}{172972800}, \quad h_{16}=\frac{1}{5881075200}, \\
h_{18} & =\frac{1}{223480857600}, \quad h_{20}=\frac{1}{9386196019200} .
\end{aligned}
$$

\section{Conclusions}

Pseudodifferential calculus is an effective tool for applying heat kernel methods to compute the terms in the expansion of a spectral action. We have used this technique to derive the terms up to $a_{12}$ in the expansion of the spectral action for the Robertson-Walker metric on a 4-dimensional geometry with a general cosmic scale factor $a(t)$. Performing the computations in Hopf coordinates, which reflects the symmetry of the space more conveniently at least from a technical point of view, we proved the independence of the integral kernel of the corresponding heat operator from three coordiantes of the space. This allowed us to furnish the proof of the conjecture of Chamseddine and Connes on rationality of the coefficients of the polynomials in $a(t)$ and its derivatives that describe the general terms $a_{2 n}$ in the expansion.

The terms up to $a_{10}$ were previously computed in [9] using their direct method, where the terms up to $a_{6}$ were checked against Gilkey's universal formulas $[17,18]$. The outcome of our computations confirms the previously computed terms. Thus, we have formed a check on the terms $a_{8}$ and $a_{10}$. In order to confirm our calculation for the term $a_{12}$, we have performed a completely different computation in spherical coordinates and checked its agreement with our calculation in Hopf coordinates. It is worth emphasizing that the high complexity of the computations, which is overcome by computer assistance, raises the need to derive the expressions at least in two different ways to ensure their validity.

We have found a formula for the coefficient of the term with the highest derivative of $a(t)$ in $a_{2 n}$ for all $n$ and make the following observation. The polynomials $Q_{2 n}$ in $a_{2 n}=Q_{2 n}\left(a(t), a^{\prime}(t), \ldots, a^{(2 n)}(t)\right) / a(t)^{2 n-3}$ are of the following form up to $Q_{12}$ :

$$
Q_{2 n}\left(x_{0}, x_{1}, \ldots, x_{2 n}\right)=\sum c_{k} x_{0}^{k_{0}} x_{1}^{k_{1}} \cdots x_{2 n}^{k_{2 n}}, \quad c_{k} \neq 0,
$$


where the summation is over all tuples of non-negative integers $k=\left(k_{0}, k_{1}, \ldots, k_{2 n}\right)$ such that either $\sum k_{j}=2 n$ while $\sum j k_{j}=2 n$, or $\sum k_{j}=2 n-2$ while $\sum j k_{j}=2 n-2$. This provides enough evidence and hope to shed more light on general structure of the terms $a_{2 n}$ by further investigations, which are under way.

\section{Acknowledgments}

We are indebted to Alain Connes for helpful discussions and encouragements on the present topic. F.F. thanks the Institut des Hautes Études Scientifiques (I.H.E.S.) and its IT department, in particular Francois Bachelier, for their support and the excellent environment and facilities during his visit in the Fall of 2013.

Open Access. This article is distributed under the terms of the Creative Commons Attribution License (CC-BY 4.0), which permits any use, distribution and reproduction in any medium, provided the original author(s) and source are credited.

\section{References}

[1] P. Amsterdamski, A.L. Berkin and D.J. O'Connor, $b_{8}$ 'Hamidew' coefficient for a scalar field, Class. Quant. Grav. 6 (1989) 1981 [INSPIRE].

[2] I.G. Avramidi, The covariant technique for the calculation of the heat kernel asymptotic expansion, Phys. Lett. B 238 (1990) 92 [INSPIRE].

[3] A.H. Chamseddine and A. Connes, Universal formula for noncommutative geometry actions: unification of gravity and the standard model, Phys. Rev. Lett. 77 (1996) 4868 [INSPIRE].

[4] A.H. Chamseddine and A. Connes, The spectral action principle, Commun. Math. Phys. 186 (1997) 731 [hep-th/9606001] [INSPIRE].

[5] A.H. Chamseddine and A. Connes, Conceptual explanation for the algebra in the noncommutative approach to the standard model, Phys. Rev. Lett. 99 (2007) 191601 [arXiv: 0706.3690] [INSPIRE].

[6] A.H. Chamseddine and A. Connes, Quantum gravity boundary terms from spectral action, Phys. Rev. Lett. 99 (2007) 071302 [arXiv: 0705.1786] [INSPIRE].

[7] A.H. Chamseddine and A. Connes, Why the standard model, J. Geom. Phys. 58 (2008) 38 [arXiv:0706.3688] [INSPIRE].

[8] A.H. Chamseddine and A. Connes, The uncanny precision of the spectral action, Commun. Math. Phys. 293 (2010) 867 [arXiv:0812.0165] [INSPIRE].

[9] A.H. Chamseddine and A. Connes, Spectral action for Robertson-Walker metrics, JHEP 10 (2012) 101 [arXiv: 1105.4637] [INSPIRE].

[10] A.H. Chamseddine, A. Connes and M. Marcolli, Gravity and the standard model with neutrino mixing, Adv. Theor. Math. Phys. 11 (2007) 991 [hep-th/0610241] [InSPIRE].

[11] A. Connes, Noncommutative geometry, Academic Press, U.S.A. (1994).

[12] A. Connes, Gravity coupled with matter and foundation of noncommutative geometry, Commun. Math. Phys. 182 (1996) 155 [hep-th/9603053] [InSPIRE]. 
[13] A. Connes, Noncommutative geometry and the standard model with neutrino mixing, JHEP 11 (2006) 081 [hep-th/0608226] [INSPIRE].

[14] A. Connes, On the spectral characterization of manifolds, J. Noncommut. Geom. 7 (2013) 1 [arXiv:0810.2088] [INSPIRE].

[15] A. Connes and M. Marcolli, Noncommutative geometry, quantum fields and motives, American Mathematical Society Colloquium Publications 55, American Mathematical Society, U.S.A. (2008).

[16] C. Estrada and M. Marcolli, Noncommutative mixmaster cosmologies, Int. J. Geom. Meth. Mod. Phys. 10 (2013) 1250086 [arXiv:1203.2679] [InSPIRE].

[17] P. Gilkey, Invariance theory, the heat equation, and the Atiyah-Singer index theorem, Mathematics Lecture Series 11, Publish or Perish Inc., Wilmington U.S.A. (1984).

[18] P. Gilkey, Asymptotic formulae in spectral geometry, Chapman \& Hall/CRC, U.K. (2004).

[19] J.M. Gracia-Bondia, B. Iochum and T. Schücker, The standard model in noncommutative geometry and fermion doubling, Phys. Lett. B 416 (1998) 123 [hep-th/9709145] [INSPIRE].

[20] B. Iochum, C. Levy and D. Vassilevich, Global and local aspects of spectral actions, J. Phys. A 45 (2012) 374020 [arXiv:1201.6637] [inSPIRE].

[21] B. Iochum, C. Levy and D. Vassilevich, Spectral action for torsion with and without boundaries, Commun. Math. Phys. 310 (2012) 367 [arXiv: 1008.3630] [InSPIRE].

[22] D. Kolodrubetz and M. Marcolli, Boundary conditions of the RGE flow in the noncommutative geometry approach to particle physics and cosmology, Phys. Lett. B 693 (2010) 166 [arXiv:1006.4000] [InSPIRE].

[23] H.B. Lawson and M.-L. Michelsohn, Spin geometry, Princeton University Press, Princeton U.S.A. (1989).

[24] M. Marcolli, Feynman motives, World Scientific Publishing Co. Pte. Ltd., Singapore (2010).

[25] M. Marcolli, Building cosmological models via noncommutative geometry, Int. J. Geom. Meth. Mod. Phys. 8 (2011) 1131 [inSPIRE].

[26] M. Marcolli and E. Pierpaoli, Early universe models from noncommutative geometry, Adv. Theor. Math. Phys. 14 (2010) 1373 [arXiv:0908.3683] [INSPIRE].

[27] M. Marcolli, E. Pierpaoli and K. Teh, The coupling of topology and inflation in noncommutative cosmology, Commun. Math. Phys. 309 (2012) 341 [arXiv:1012.0780] [INSPIRE].

[28] M. Marcolli, E. Pierpaoli and K. Teh, The spectral action and cosmic topology, Commun. Math. Phys. 304 (2011) 125 [arXiv: 1005.2256] [INSPIRE].

[29] W. Nelson, J. Ochoa and M. Sakellariadou, Constraining the noncommutative spectral action via astrophysical observations, Phys. Rev. Lett. 105 (2010) 101602 [arXiv:1005.4279] [INSPIRE].

[30] W. Nelson and M. Sakellariadou, Natural inflation mechanism in asymptotic noncommutative geometry, Phys. Lett. B 680 (2009) 263 [arXiv:0903.1520] [INSPIRE].

[31] W. Nelson and M. Sakellariadou, Cosmology and the noncommutative approach to the standard model, Phys. Rev. D 81 (2010) 085038 [arXiv: 0812.1657] [INSPIRE]. 
[32] A. Sitarz, Spectral action and neutrino mass, Europhys. Lett. 86 (2009) 10007 [arXiv:0808.4127] [INSPIRE].

[33] W.D. van Suijlekom, Renormalization of the spectral action for the Yang-Mills system, JHEP 03 (2011) 146 [arXiv:1101.4804] [INSPIRE].

[34] W.D. van Suijlekom, Renormalization of the asymptotically expanded Yang-Mills spectral action, Commun. Math. Phys. 312 (2012) 883 [arXiv:1104.5199] [INSPIRE].

[35] A.E.M. van de Ven, Index free heat kernel coefficients, Class. Quant. Grav. 15 (1998) 2311 [hep-th/9708152] [INSPIRE]. 